\title{
Pictorial depth and the Poggendorff illusion
}

\author{
THEODORE E. PARKS and LUMEI HUI \\ University of California, Davis, California
}

\begin{abstract}
An informal demonstration is offered, which strongly supports previous contentions that, when the elements of a Poggendorff display appear to be arranged in pictorial space such that the two critical line segments are at different heights, an illusory impression of misalignment may occur. A second pair of demonstrations shows, however, that such a height difference is neither a necessary nor a sufficient cause of the illusion. In addition, the harmful effect of adding certain pictorial elements to the standard Poggendorff pattern requires a new understanding.
\end{abstract}

The well-known Poggendorff illusion-which is to say, the apparent misalignment of the two short diagonal line segments in patterns such as that in Figure 1a-has been the object of a number of possible explanations, more than one of which may be true (see, for example, the discussion in Rock, 1975, and Day \& Kasperczyk, 1985). Here, only one of these theories will be considered in any explicit detail: the possibility that a pictorial height difference that is suggested by the elements of the pattern is responsible for the illusory misalignment that occurs there (Gillam, 1971, 1980). It will be demonstrated that such pictorial factors can indeed be so powerful as to be accompanied by erroneous impressions of misalignment, even under what would seem to be extremely disadvantageous circumstances for the occurrence of that illusion. However, it will then be argued that such pictorial factors do not cause the illusion.

\section{Depth and Misalignment Effects}

As Gillam (1971, 1980) pointed out, the elements of Figure 1a may be elaborated so that the vertical elements appear to be two edges of a vertical surface, such as the end of a box, whereas an additional set of four radiating lines appears to comprise the edges of the sides of that box (see Figure 1b). The two original diagonal segments will then be seen to be misaligned within that threedimensional arrangement, the one being "higher" on its wall than the other is on its wall (or at different lateral locations when the figure is rotated $90^{\circ}$ ). Perhaps, Gillam suggested, the unelaborated pattern (Figure 1a) is perceived in the same way, and the resulting misalignment in pictorial space is inadvertently perceived as (is confused with) a misalignment in the two-dimensional pattern itself. ${ }^{1}$ Moreover, she went on to strengthen the attractiveness of this possibility with numerous corollary demonstrations that other elaborations, which provide other arrangements in pictorial depth, will reduce the illusion. For instance, little if any illusory misalignment is apparent in Figure 1c. This result is consistent with her

Correspondence may be addresed to Theodore E. Parks, Department of Psychology, University of California, Davis, California 95616. proposal insofar as the pictorial slant of the central surface tends to capture the line segments so as to make them appear to lie in the same receding imaginary plane as the rectangle and-what is more important-in the same plane as each other. Moreover, if they are seen thus, they will also be seen to lie at the same height on that plane, and so no basis for a confused impression of misalignment will be present.

Indeed, additional striking demonstrations of the importance of an apparent arrangement in space can be devised. To cite a new one, with careful effort it will be noticed that, when the diagonal stripe in Figure $2 a$ is seen as a picture of a stripe of paint which starts across one step, swerves across the vertical surface, and then continues straight along the next, there will be a subtle, but insistent, impression that the two end segments are not aligned two-dimensionally, either. (It should be noted that it is very important that the appearance of a threedimensional "swerve" be carefully maintained while the latter observation is being made, a point that will be emphasized later.)

Thus, the Poggendorff illusion can survive what would intuitively seem to be a very important alteration of the usual pattern: the actual connection of the usually separate segments. In fact, with practice, the same can be said for Figure $2 b$, this being, of course, somewhat closer to the classic pattern. Finally, even that classic pattern itself can be seen to represent elements pictorially on two vertically separated horizontal planes, with the intervening vertical plane and the middle segment of the line omitted (Figure 2c).

On the other hand (as the reader has probably noticed already), if the stripe in Figure 2a is seen not as one that bends in order to lie successively on each of the three depicted surfaces, but as a straight strip of rigid material lying above them, the illusion disappears. The same thing happens if the diagonal line in Figure $2 b$ appears to be a straight rod receding in pictorial space.

Apparently, then, an impression of a three-dimensional "swerve," and only such an impression, is accompanied by an impression of two-dimensional misalignment, even to the extent that the impression of misalignment over- 


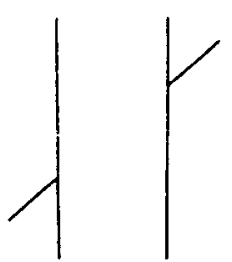

a

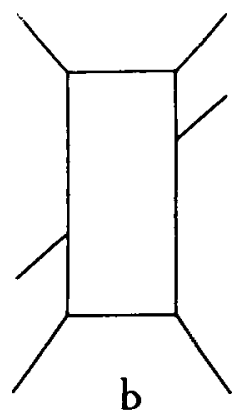

b

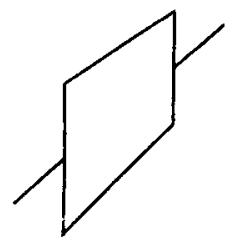

C

Figure 1. An example (a) of the classic Pogendorfi pattern, along with variations (b and c) on two elaborations of that pattern which were presented by Gillam (1971).

comes the fact that pairs of end segments are drawn as parts of continuous straight lines. But such a correlation does not-as correlations never do-prove causation.

\section{Toward an Understanding}

An alternative understanding of the relationship between pictorial depth and the Poggendorff illusion may begin with an effect reported by Kennedy (1987): Just as the pictorial arrangement present in Figure $1 \mathrm{c}$ reduces the illusion, so does holding a standard pattern at a slant and rotating it such that the observer sights down the nearer segment toward the farther one. The result of such "sighting down" can easily be appreciated if, for example, one views Figure 1a from the lower left at a place more or less in line with the diagonal segments.

The essential point is that these two procedures-certain pictorial contexts and sighting down the two segmentsmight have more in common than the mere fact that they both reduce the illusion. That is, reconsideration of Figure 1c reveals that the geometry of the pictorial arrangement in space seen there is such that the viewer is pictorially in a position that approximates sighting down one segment toward the other. And it may be that this pictorial representation of the experience of sighting down produces the same effect as does the actual act itself.

To take the other side of this coin, the point of displays in which a pictorial arrangement in depth is accompanied by illusory nonalignment may be that such an arrangement prevents pictorial sighting down and therefore allows the illusion to persist. ${ }^{2}$ If so, it should be possible to devise displays in which pictorial depth is present and in which illusory misalignment also occurs, but this time solely because of excluding the possibility of sighting down.

Figure 3 shows that this is in fact the case. Pictorially, this pattern is meant to represent two segments at equal height arranged in parallel, one of them being at a greater distance. The very important lesson here is that, despite the suggestion of equal heights, the line segments appear to be subtly, but noticeably, misaligned in the vertical dimension. Thus, the illusion occurs, but this time not because of a difference in pictorial height; no such difference is suggested within this pattern.

To put it differently, the results of viewing Figure 4 both support and contradict Gillam's demonstration, a paradox that might be clarified by means of comparison with Figure 1b. In the version of Gillam's pattern shown

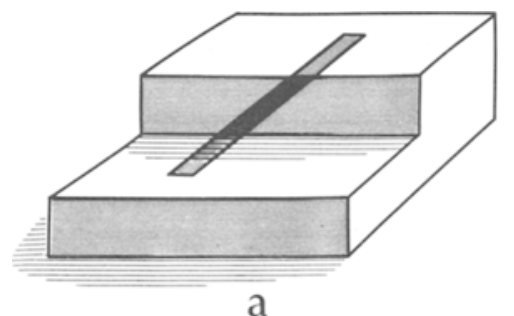

a
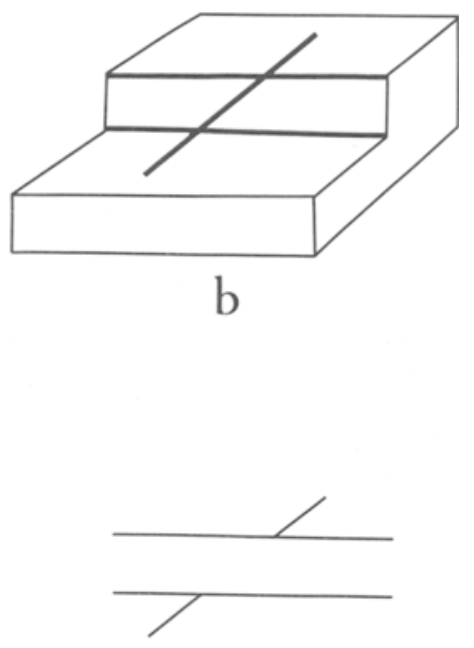

C

Figure 2. A new elaboration of the classic pattern (a), which is simplified (b), finally to the point of becoming the classic pattern rotated $90^{\circ}$ (c). 


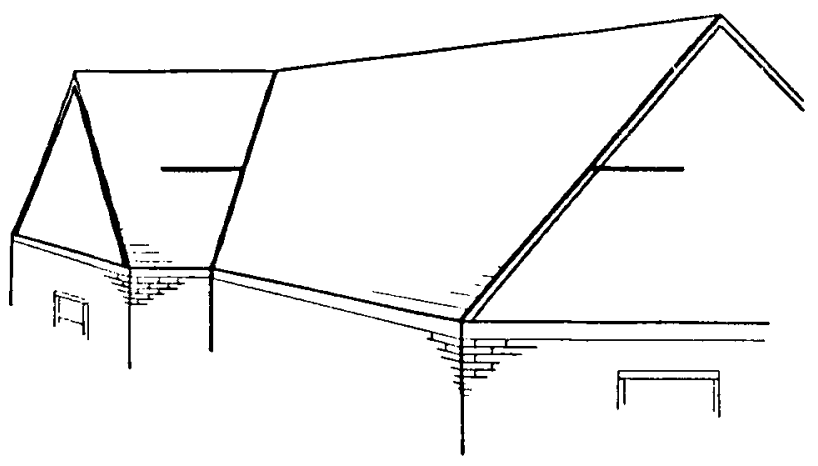

Figure 3. A pattern in which the segments appear to be at the same height in pictorial space, but in which the illusion may occur anyway.

in Figure 1b, one of the two critical segments is seen to be higher than the other within the three-dimensional space pictorially represented there. Since corresponding illusory misalignment is experienced, it would be quite reasonable to assume that it might be due to the perceived height difference. However, in Figure 3, the segments also appear to be misaligned, even though in this case there is no corresponding pictorial reason for them to be seen at different heights. Rather, they are at the same suggested height, so such a pictorial difference cannot possibly be a necessary cause of the illusory effect. On the other hand, what both of these patterns do have in common is that in neither case do the critical segments appear to be positioned such that pictorial sighting down is possible. And the absence of that possibility might be critically important to their mutual effectiveness.

But if a pictorial difference in height is not a necessary cause of the illusion, is it even a sufficient cause? Figure 4 argues against even this weaker version of the theory, in that the segments seen there, though clearly at different heights, show very little tendency toward illusory misalignment (especially if a three-dimensional appearance is carefully maintained).

Indeed, when a group of 24 naive observers was individually shown a version of Figure 4 in which the upper segment was movable and controlled by them, and they were asked to align the two segments, they produced a mean error of only $.13 \mathrm{~cm}$ (or $.03^{\circ}$ of visual arc), which is a distance less than the thickness of the lines themselves. By contrast, when these same subjects were shown a control pattern in which the Poggendorff pattern was rotated $90^{\circ}$ clockwise on the pictorial surface of the roof, they produced a reliably larger mean error of $.30 \mathrm{~cm}[t(23)$ $=3.53, p<.01] .^{3}$

Apparently, then, although patterns that yield a reduced illusion may include a suggestion that the critical segments are at the same height (e.g., Figure 1c), in other cases they do not (e.g., Figure 4). On the other hand, what both of these patterns do have in common is an approximation of the situation of sighting down one segment toward the other. For instance, Figure 4 very obviously represents the situation that occurs when a standard pattern is tilted away and viewed from one corner. Similarly, the two critical segments of Figure 1c appear as they would if a standard pattern were to be rotated about the vertical axis and twisted so that those segments became parallel to the earth (and, as a result, became closer to alignment with the line of sight). Perhaps, then, the lesson of displays that do not produce a strong illusion is that, in them, true alignment is made more evident by pictorial sighting down, thereby resisting illusory misalignment.

\section{Conclusions and Questions}

In summary, the significance of Gillam's $(1971,1980)$ demonstrations apparently lies in her delineation of the circumstances that both allow and do not allow illusory misalignment to go unchallenged. As noted before, what the cause or causes of that illusion might be is beyond the purpose of the present paper, but we are left with a new and equally intriguing question: How can the mere pictorial stance of looking down one segment at another produce the beneficial (anti-illusory) effect of actually doing so? After all, actually sighting down one segment toward another would be expected to reduce the illusion by, at the very least, reducing the retinal size of the gap between those two segments. But mere pictorial sighting does not alter the retinal image at all.

Of course, introducing an impression of slant can alter the experience that arises from any particular retinal image. For example, an ellipse is the retinal projection of a circular shape at a slant to the line of sight, and an elliptical shape in a painting will be perceived as representing a circularly shaped thing when that thing is perceived to be at a slant. By the same token, two aligned line segments in the retinal image are the proper projection of two aligned segments on a receding plane and may be perceived as such.

But all of that argument begs the issue. In the case of the ellipse, only perceived shape is altered when apparent slant is introduced. For instance, if an ellipse represents a wheel rim with two spokes attached to it opposite to each other, the alignment of those spokes would

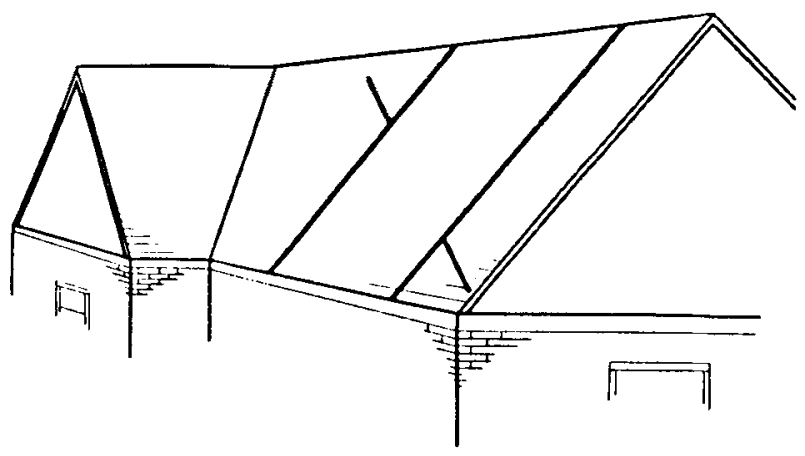

Figure 4. A pattern in which the critical segments appear to be at different heights in pictorial space, but do not appear to be misaligned much if at all. 
presumably be apparent if either the elliptical shape or the pictorial receding circle were perceived. In a Poggendorff display, however, more than the appearance of each isolated segment is altered when the segments appear to be receding, since then, and only then, may those segments appear (correctly) to be aligned. To put it another way: accuracy is introduced that goes beyond the accurate perception of individual shapes. Our response to pictorial perspective also tends to destroy the illusion. Therein remains the mystery: Why does a merely pictorial opportunity to "sight down" work so well?

\section{REFERENCES}

DAY, R. H., \& KASPERCZYK, R. T. (1985). Apparent displacement of lines and dots in a parallel-line figure: A clue to the basis of the Poggendorff effect. Perception \& Psychophysics, 38, 74-80.

GilLAM, B. (1971). A depth processing theory of the Poggendorff illusion. Perception \& Psychophysics, 10, 211-216.

Gillam, B. (1980). Geometric illusions. Scientific American, 242, 102-111.

Green, R. T., \& HoYle, E. M. (1964). The influence of spatial orientation on the Poggendorff illusion. Acta Psychologica, 22, 348-366.
KENNEDY, J. M. (1987). Lo, perception abhors not a contradiction. In S. Petry \& G. H. Meyer (Eds.), The perception of illusory contours (pp. 253-261). New York: Springer-Verlag.

Rock, I. (1975). An introduction to perception. New York: Macmillan.

\section{NOTES}

1. Previously, Green and Hoyle (1964) had also presented a figure very similar to the present Figure $\mathrm{lb}$, but their discussion of it was somewhat different.

2. It cannot be overemphasized that the discussion that follows is not intended to explain why the Poggendorff illusion occurs (when it does occur). The goal, instead, is to seek to understand why that illusion, under some conditions, either does not occur or occurs in a greatly reduced form. Thus, to use Kennedy's example, no one would claim that not being allowed to physically sight down a traditional display "causes" the illusory misalignment: the illusion is clearly caused by some other factor or factors which, whatever they may be, are less effective if physical sighting down is allowed. The same is assumed to be true for pictorial sighting down.

3. The patterns were approximately $20 \mathrm{~cm}$ in overall height and were viewed from a distance of $2.5 \mathrm{~m}$. Each subject made four settings of each display in balanced order, each trial starting with the movable segment randomly to one side or the other at a position which was obviously misaligned.

(Manuscript received October 3, 1988; revision accepted for publication May 15, 1989.) 